\title{
NOTA SOBRE A PRESENÇA DA LUTZOMYIA LONGIPALPIS (LUTZ \& NEIVA, 1912) NA ILHA GRANDE, ESTADO DO RIO DE JANEIRO, BRASIL*
}

\author{
Nelson A. Araújo Filho** e lítalo A. Sherlock ***
}

\begin{abstract}
Foi relatado o encontro pela primeira vez, de Lutzomyia longipalpis na Praia Vermelha, municipio de Angra dos Reis, Rio de Janeiro, e chamada atenção do fato em relação à possivel conotação em Leishmania Visceral no futuro, tal qual, ocorreu em Bangu e Jacarepaguá no Rio de Janeiro.
\end{abstract}

\section{INTRODUÇÃO}

A presença da Lutzomyia longipalpis no Estado do Rio de Janeiro foi descrita incialmente por Lutz \& Neiva, 1912, a partir de um exemplar fêmea capturado no município de Mangaratiba; entretanto, este achado foi posto em dúvida por alguns autores $\left(\right.$ Lima $^{8}$, Barreto $^{3,4}$ ). Até recentemente era desconhecida a real prevalência da espécie no Estado do Rio de Janeiro. A ocorrência da L. longipalpis é predominante nas áreas de calazar do país, como assinalam Deane ${ }^{5}$, Mangabeira Filho9, Martins \& Cols $^{10}$ e Sherlock \& Guitton ${ }^{12}$.

Em 1974, na eclosão da epidemia de Leishmaniose tegumentar americana, em Jacarepaguá, Estado do Rio de Janeiro, foram coletados cinco exemplares da L. longipalpis (FIOCRUZ ${ }^{6}$ ).

No mês de setembro de 1977 um caso autóctone de calazar humano foi registrado no bairro de Bangu, área suburbana do Estado do Rio de Janeiro. Na procura do vetor alado foram capturados 1585 flebotomíneos, sendo a $L$. longipalpis a segunda espécie mais coletada, com 684 exemplares (Sobroza \& Cols ${ }^{1}$ ).

Em estudos realizados durante os anos de 1976/1977 na localidade de Praia Vermelha, Ilha Grande, município de Angra dos Reis, Estado do Rio de Janeiro, a cerca de um surto de
Leishmaniose tegumentar americana, investigou-se também a presença dos transmissores dessa zoonose na área (Araújo Filho \& Cols $^{1}$, Araújo Filho ${ }^{2}$ ).

\section{MATERIAL E MÉTODOS}

Nas pesquisas efetuadas durante o período de fevereiro de 1976 a abril de 1977 foram realizadas capturas em domicílios, galinheiros abrigos naturais, tendo-se, ainda, utilizado animais domésticos e silvestres como iscas. Coletou-se um total de 4.192 flebotomineos; as espécies de maior prevalência sendo Lutzomyia intermedia e Lutzomyia migonei. Em três galinheiros e um domicilio foram coletados 43 exemplares da L. longipalpis (Fig. 1), sendo 36 machos e sete fêmeas, representando a terceira espécie mais coletada na área.

Apesar de não haver sido relatada a presença de Calazar autóctone naquela área de trabalho e sendo atualmente conhecida apenas a ocorrência de Leishmaniose tegumentar americana (Araújo Filho \& Cols ${ }^{1}$, Araújo Filho ${ }^{2}$ ) o encontro da $L$. longipalpis demonstra a importância que este fato pode vir a ter sob o ponto de vista epidemiológico.

- Trabalho do Departamento de Medicina Preventiva da Faculdade de Medicina da Universidade Federal do Rio de Janeiro, realizado com auxilio financeiro do CNPq.

* Docente da Universidade do Amazonas, Mestre em Doenças Infecciosas e Parasitárias pela Universidade Fede ral do Rio de Janeiro.

* * Pesquisador Titular do Centro de Pesquisas Goncalo Moniz/BA - (FIOCRUZ). 


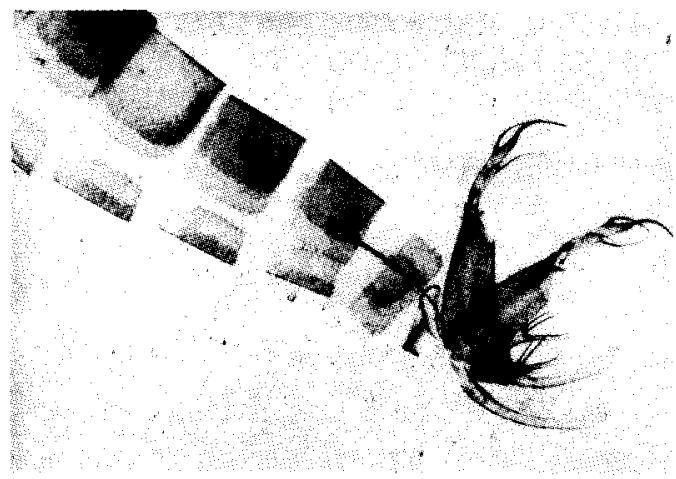

Fig. 1 - Lutzomyia longipalpis - genitália masculina.

Forattini \& $\mathrm{Cols}^{7}$ em 1971 registram a presença de 19 exemplares da L. longipalpis, no bairro do Arado, município do Salto do Pirapora, Estado de São Paulo. Os autores chamam atenção das autoridades sanitárias do Estado para tal fato.

O encontro da L. longipalpis, no Estado de São Paulo, e atualmente nas duas localidades referidas no Estado do Rio de Janeiro, assume elevada importância em termos de saúde pública. Sabe-se que migrações internas para o Estado do Rio de Janeiro são uma constante, principalmente de populações procedentes de áreas de Calazar do país. A ocorrência da forma inaparente do Calazar e mesmo de casos clinicamente estabelecidos pode provocar a disseminação da doença no Estado do Rio de Janeiro, pois a presença do vetor alado já poderá manter uma epidemia de conseqüências graves, levando-se em consideração que o nível imunitário da população do Estado do Rio de Janeiro provavelmente é baixo ou quase nulo para o $\mathrm{Ca}$ lazar.

Um sistema de vigilância sanitária deve ser orientado para o diagnóstico do Calazar, do contrário, o Estado do Rio de Janeiro, poderá registrar mais uma doença de prognóstico grave e muitas vezes fatal no rol das endemias transmitidas por vetores.

\section{AGRADECIMENTOS}

Os autores agradecem a colaboração da Dra. Neide Guitton e à técnica de entomologia Célia Maria Dias Lima, do Núcleo de Pesquisas da Bahia - FIOCRUZ, pela preparação e taxonomia dos flebotomíneos deste trabalho.

\section{SUMMARY}

For the first time it has been detetect Lutzomyia longipalpis at Praia Vermelha, a beach of an offshore island of the Rio de Janeiro State (IIha Grande), Brazil and attention must be paid to its possible epidemiological importance.

\section{REFERÊNCIAS BIBLIOGRÁFICAS}

1. ARAÜJO FILHO, N.A.; WANKE, B.; COUTINHO, S.G. \& COURA, J.R. (1976) Surto de Leishmaniose tegumentar americana na Ilha Grande. In. Cong. Soc. Bras. Med. Trop., 12\%, Belém.

2. ARAÚJO FILHO, N.A. Epidemiologia da Leishmaniose tegumentar americana na llha Grande, Rio de Janeiro. Estudos sobre a infecção humana, reservatórios e transmissores. Tese de Mestrado, Pós-Graduação em doenças infecciosas e parasitárias. Fac. Med. da Universidade Federal do Rio de Janeiro, Rio de Janeiro, $148 \mathrm{p}$.

3. BARRETO, M.P. Catálogo dos flebotomineos americanos. Arq. Zool. São Paulo, 5: 177-242, 1947.

4. BARRETO, M.P. Nova contribuição para o estudo da distribuição geográfica dos flebótomos americanos (Diptera, Psychodidae) Arq. Hig. e Saúde Públ. São Paulo, 13: 211-226. 1950.

5. DEANE, L.M. Leishmaniose visceral no Brasil. Estudos sobre reservatórios e transmissores realizados no Estado do Ceará. Serviço Nacional de Educação Sanitária, Rio de Janeiro, 162 p. 1956.

6. FUNDAÇÃO OSWALDO CRUZ (FIOCRUZ) Relatório do grupo de trabalho coordenador das atividades de estudo e controle da Leishmaniose tegumentar americana na área de atuação do posto Samuel Libânio (Jacarepaguá, Rio de Janeiro), 25 p. 1974.

7. FORATTINI, O.P.; RABELLO, E.X. \& PATOLLI, D. Sobre o encontro da Lutzomyia longipalpis (Lutz \& Neiva, 1912) no Estado de São Paulo, Brasil. Rev. Saúde Públ. São Paulo, 4: 99-100, 1971.

8. LIMA, A.M. da COSTA. Flebótomos americanos (Diptera, Psychodidae). Mem. Inst. Oswaldo Cruz, 26: 15-69.

9. MANGABEIRA FILHO, O. Sobre a siste 
mática e biologia dos flebótomos do Ceará. Rev. Bras. Malariol., 21 : 3-26, 1969.

10. MARTINS, A.V.; BRENER, Z.; MOURÃO, D.G.; LIMA, M.M.; SOUZA, M.A. \& SILVA, J.E. da - Calazar autóctone em Minas Gerais. Rev. Bras. Malariol., 8: 554-563, 1956.

11. SABROZA, P.C.; SOUZA, M.A. \& MAR-
ZOCHI, M.C.A. Flebotomíneos na Cidade do Rio de Janeiro. In. Cong. Bras. Med. Trop., 140 João Pessoa, 1978.

12. SHERLOCK, I.A. \& GUITTON, N. Observações sobre o Calazar em Jacobina, Bahia. Il Alguns dados sobre o Phlebotomus longipalpis, o principal transmissor. Rev. Bras. Malariol., 21 : 541-548. 